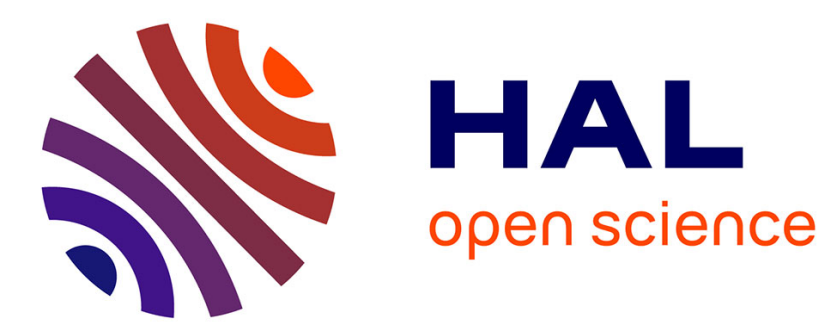

\title{
The Trajectory approach for AFDX FIFO networks revisited and corrected
}

\author{
Xiaoting Li, Olivier Cros, Laurent George
}

\section{To cite this version:}

Xiaoting Li, Olivier Cros, Laurent George. The Trajectory approach for AFDX FIFO networks revisited and corrected. The 20th IEEE International Conference on Embedded and Real-Time Computing Systems and Applications, IEEE, Aug 2014, Chongqing, China. hal-00975730

\section{HAL Id: hal-00975730 \\ https://hal.science/hal-00975730}

Submitted on 9 Apr 2014

HAL is a multi-disciplinary open access archive for the deposit and dissemination of scientific research documents, whether they are published or not. The documents may come from teaching and research institutions in France or abroad, or from public or private research centers.
L'archive ouverte pluridisciplinaire HAL, est destinée au dépôt et à la diffusion de documents scientifiques de niveau recherche, publiés ou non, émanant des établissements d'enseignement et de recherche français ou étrangers, des laboratoires publics ou privés. 


\section{The Trajectory approach for AFDX FIFO networks revisited and corrected}

\author{
Xiaoting LI, Olivier CROS \\ ECE Paris - LACSC \\ 37, quai de Grenelle \\ 75015 Paris, France \\ Email: $\{$ xiaoting.li,cros\}@ece.fr
}

\author{
Laurent GEORGE \\ Université Paris-Est / LIGM \\ Bat Copernic - 5, bd Descartes \\ 77454 Champs sur Marne, France \\ Email: lgeorge@ieee.org
}

\begin{abstract}
We consider the problem of dimensioning realtime AFDX FIFO networks with a worst-case end-to-end delay analysis. The state-of-the-art has considered several approaches to compute these worst-case end-to-end delays. Among them, the Trajectory approach has received more attention as it has been shown to provide tight end-to-end delay upper bounds. Recently, it has been proved that current Trajectory analysis can be optimistic for some corner cases, leading in its current form, to certification issues. In this paper, we first characterize the source of optimism in the Trajectory approach on detailed examples. Then, we provide a correction to the identified problems. Two problems are solved: the first one is on the definition of the time interval to consider for the worst-case end-to-end response time computation of flows at their source nodes. The second one is on the way that serialized frames are taken into account in the worst-case delay analysis.
\end{abstract}

\section{INTRODUCTION}

Reliability and safety are primary constraints in highcritical industrial systems such as aircraft and public transport systems. Furthermore, the needs in these systems are drastically increasing with the development of intelligent transport systems : in addition to safety requirements, we want to add comfort, usability, information management, etc.. This increase in terms of functionalities implies to exchange more and more data and resources through networks. That is why we need specific network architectures that can manage this workload.

High-critical real-time systems often rely on deterministic network architectures to exchange information between endsystems. When throughput is under concern, switched Ethernet networks are more and more considered. Several solutions have been proposed like Synchronous Ethernet (SyncE) [1], Avionics Full DupleX switched Ethernet (AFDX) [2] , Flexible Time Triggered Switched Ethernet (FTT-SE) or Time Triggered Ethernet (TTEthernet) [3]. They are network architectures which are able to bound, with clock synchronization (SyncE, FTT-SE and TTEthernet) or without clock synchronization (AFDX), the end-to-end delay of a message sent through the network, In order to guarantee deterministic communications, several worst-case analysis have been proposed to compute the upper-bounds of the end-to-end communication delays of all the flows sent in the network, and to assure their reliability.

There exist different approaches for computing end-to-end delays in real-time networks. Among them, we can cite:

- The Holistic approach, is a pessimistic way (showed in [4]), and more considered as a general approach [5].
It consists of computing end-to-end delay bounds by considering a worst-case scenario (possibly unreachable) in each node visited by a flow.

- $\quad$ The Network Calculus approach [6], [7], which considers worst-case scenario of a flow at each visited node according to service curves. This approach has been improved by considering the serialization effect by [8].

- The Trajectory approach [9], [10], [11], [4], which consists in representing the network not just as a set of nodes, but as a set of flow trajectories through nodes and where each trajectory (associated to one flow) is a potential source of delay for the other flows. This approach is based on the computation of end-to-end delays induced by other frames in the network, and specially the one induced by their serialization on the input ports of switches (called the serialization delay). It was shown that the classical Trajectory approach provide tighter upper bounds than the holistic one but can be pessimistic. Its pessimism has been analyzed in [12].

In [13], [8], the Trajectory approach has been improved by taking into account the serialization of frames sent on the same nodes (called serialization effect in the following). Bauer\&al. has shown interesting properties of worst-case delay analysis in the avionics context as it can bring slightly more accurate delay upper bounds than the classical Network Calculus approach. In this work, we focus on the Trajectory approach.

More recently in [14], a counter-example has been given, showing that in its current form the Trajectory approach can bring optimistic results for corner cases. A discussion about the source of pessimism has been given in [14], while no solution has been proposed.

The main objective of this paper is to identify the source of optimism in the Trajectory approach and to propose a solution to it. The first contribution of our work is to characterize this optimism on a detailed example with a detailed analysis. Then, we give a correction of the optimism problem first on the considered example and then by formalizing a correction in the general case. We show that the error rate of the state-ofthe-art Trajectory approach w.r.t. our is higher than $10 \%$ in the examples we considered.

The paper is organized as follows. II introduces the network 
model and flow model considered in the paper. An real-time AFDX network is presented. III presents the classical Trajectory approach and its improvement with the serialization effect. IV shows a counter-example where the Trajectory approach introduces optimism in the computation. $\mathrm{V}$ demonstrates the reason of this optimism and proposes a correction to the identified problems in the Trajectory approach. VI concludes the paper.

\section{REAL-TIME AFDX NETWORK}

Avionics Full DupleX Switched Ethernet (AFDX) [2] is a switched Ethernet network which has been defined for the avionics context and developed for modern aircraft such as Airbus A380. AFDX is one of the industrial applications of real-time switched Ethernet networks. The descriptions of the network and flow models are given in the following paragraphs.

\section{A. Network model}

In this paper, we study a real-time AFDX switched Ethernet network which is a network able to provide a deterministic data transmission service, through an Ethernet layer. The inputs and outputs of the network are source nodes, called End Systems $(E S)$ in the context of AFDX network. These source nodes are interconnected by a full duplex switched Ethernet. We consider a homogeneous single network.

Each source node sends a set of flows through an output port with a buffer supporting First In First Out (FIFO) scheduling. It can be connected to only one port of a switch and each port of a switch can be connected to at most one node. Traffic shaping technique is adopted at the output port of each source node in order to guarantee a minimum interval time between two consecutive frames of a flow (also called gap in AFDX).

Each switch uses a store and forward policy. It has one buffer at each output port which supports the FIFO scheduling. It receives frames from input ports and forwards them to the corresponding output ports based on a static routing table. There is a switching latency (technological latency) to deal with the frame forwarding between an input port and an output port of a given switch and it is upper bounded by a known value $s l$.

Links between switches are full-duplex, which guarantees no collisions on links. The bandwidth (transmission rate) of the network is denoted by $R$.

A classical AFDX architecture is depicted in Figure 1. It includes six End Systems $E S_{1}, E S_{2}, E S_{3}, E S_{4}, E S_{5}$ and $E S_{6}$ interconnected by three switches $S_{1}, S_{2}$ and $S_{3}$ via fullduplex links.

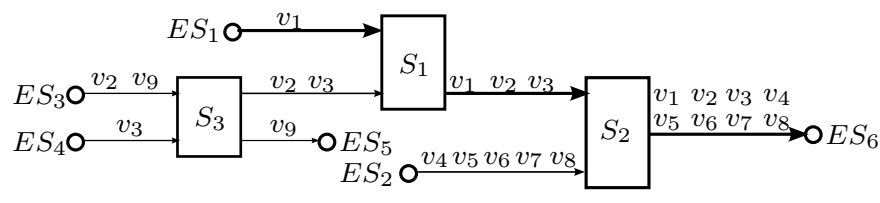

Fig. 1. An illustrative AFDX network example

\section{B. Flow model}

A Virtual Link $(V L)$ standardized by ARINC-664 is a concept of virtual unidirectional communication channel. Each VL flow $v_{i}$ transmitted over an AFDX network is characterized by the Bandwidth Allocation Gap $\left(B A G_{i}\right)$, which is the minimum duration between two consecutive frames of $v_{i}$, as well as $l_{\text {mini }_{i}}$ and $l_{\text {max }_{i}}$, which are the minimum and maximum frame lengths.

In this paper, we assume that $n$ VL flows $v_{i}, i \in$ $\{1,2, \ldots, n\}$ are transmitted over the AFDX network in order to exchange data. A VL flow $v_{i}$ has a path defined by a sequence of output ports $\mathcal{P}_{i}=\left\{\right.$ first $_{i}, \ldots$, last $\left._{i}\right\}$, where the first $_{i}$ is the source node of flow $v_{i}$ and last $_{i}$ is the last visited output port of flow $v_{i}$ along the path $\mathcal{P}_{i}$. For instance, $v_{1}$ in Figure 1 follows the path $\mathcal{P}_{1}=\left\{E S_{1}, S_{1}, S_{2}\right\}$. Its source node is first $_{1}=E S_{1}$. Its last visited output port is last $t_{1}=S_{2}$. This flow model can be extended to multicast flows where each path is associated to one receiver. For the sake of simplicity, we only consider unicast flows in this paper.

Sporadic VL flows are transmitted over the network. The temporal features of a given VL flow $v_{i}$ are defined by the following parameters:

- the minimum inter-frame duration $T_{i}$, which corresponds to the value of $B A G_{i}$ associated to flow $v_{i}$, and

- the worst-case transmission time (WCTT) of one frame $f_{i}$ of flow $v_{i}$. It is denoted by $C_{i}$ and can be computed by $C_{i}=\frac{l_{\max _{i}}}{R}$. Indeed, instead of defining a specific WCTT for each node and each frame, we define the worst-case transmission time of a frame transmitted at all nodes.

In this paper, for the sake of simplicity, we do not consider release jitter for each flow, but a release jitter can be easily taken into account in the computation of worst case end-to-end delays.

\section{UNDERSTANDING THE TRAJECTORY APPROACH}

The Trajectory approach allows us to compute a bound on the worst-case transmission delay of any flow transmitted on a switched Ethernet network. It has been first proposed for the First In First Out (FIFO) scheduling in [9] and extended for non-preemptive Fixed Priority (FP) scheduling in [10], [11]. In the following paragraphs, we first recall the classical Trajectory approach principles. Then, we present the integration of serialization effect in the Trajectory approach proposed in the state-of-the-art.

\section{A. Notations}

For a flow $v_{i}$ following a path $\mathcal{P}_{i}=\left\{\right.$ first $_{i}, \ldots$, last $\left._{i}\right\}$, we focus on a frame $f i$ which arrives at the output port of first $_{i}$ at time $t$. The following notations are given for the computation.

- $\quad h$ represents any output port of a node or of a switch in our network.

- $h+1$ (resp. $h-1$ ) represents the previous (resp. following) output port of the output port $h$. 
- $b p^{h}$ represents the busy period of frame $f i$ at the output $h$. A busy period means a time interval during which there is no idle time.

- $F T_{\min }^{h}$ is the smallest WCTT among the frames transmitted during $b p^{h}$. It is computed by $F T_{m i n}^{h}=$ $\min _{h \in \mathcal{P}_{j}}\left(C_{j}\right)$.

- $\quad M_{i}^{h}$ is considered as the earliest arrival time of the first packet that will delay frame $f_{i}$ on the output port $h$. It is computed by:

$$
M_{i}^{h}=\sum_{k=\text { first }_{i}}^{h-1}\left(F T_{\text {min }}^{k}+s l\right)
$$

- $\quad S_{\text {max }_{i}}^{h}$ and $S_{\text {min }_{i}}^{h}$ are the maximum and the minimum delay experienced by frame $f_{i}$ from its source node first $_{i}$ to the output port $h$.

- $\quad t$ is the arrival time of a frame under study $f_{i}$ at the input port of node first $_{i}$. This arrival time should be considered w.r.t. a reference time 0 , which is our time origin. Indeed, in the architectures we want to focus on, frames that do not necessarily arrive at time 0 , but can be delayed and arrive later.

- We define a time interval used to compute the maximal number of frames about to delay our focused message, $A_{i, j}$, as defined in [14]

$$
A_{i, j}=\operatorname{Smax}_{i}^{h}-\operatorname{Smin}_{j}^{h}-M_{i}^{h}+\operatorname{Smax}_{j}^{h}
$$

- $(a)^{+}=\max \{a, 0\}$.

Let's illustrate these definitions by an example. We want to study the following elementary network

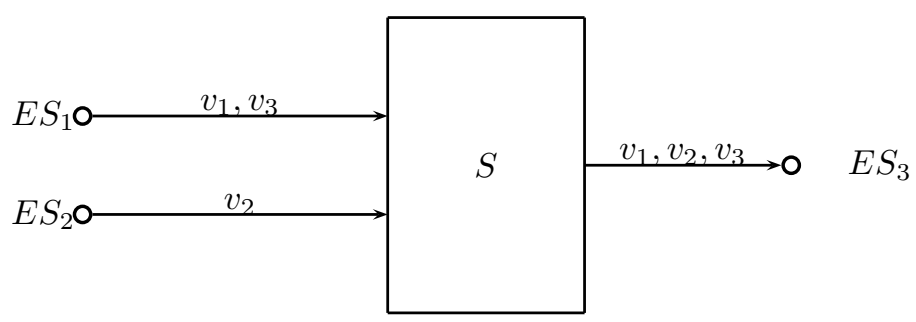

Fig. 2. Understanding the standard notations for the trajectory approach, given three flows $v_{1}, v_{2}, v_{3}$ arriving on the same switch $S$

We consider that the first input port of $S$ receives two different flows, $v_{1}$ and $v_{3} . v_{1}$ emits a frame $f_{1}$, with $C_{1}=30$ and $T_{1}=4000$, and $v_{3}$ sends $f_{3}$, with $C_{3}=20$ and $T_{3}=4000$. Following the same way, the flow $v_{2}$ sends only one packet through the network, called $f_{2}$, with $C_{2}=40$ and $T_{2}=90$. We want to focus on the packet $f_{3}$, which arrives on $E S_{1}$ at time $t=20$. Considering this system, we can represent its behavior with the figure 3 .

\section{B. Calculating an end-to-end delay}

Actually, the computation of the Trajectory approach in FIFO context is summarized by the combination of 4 different
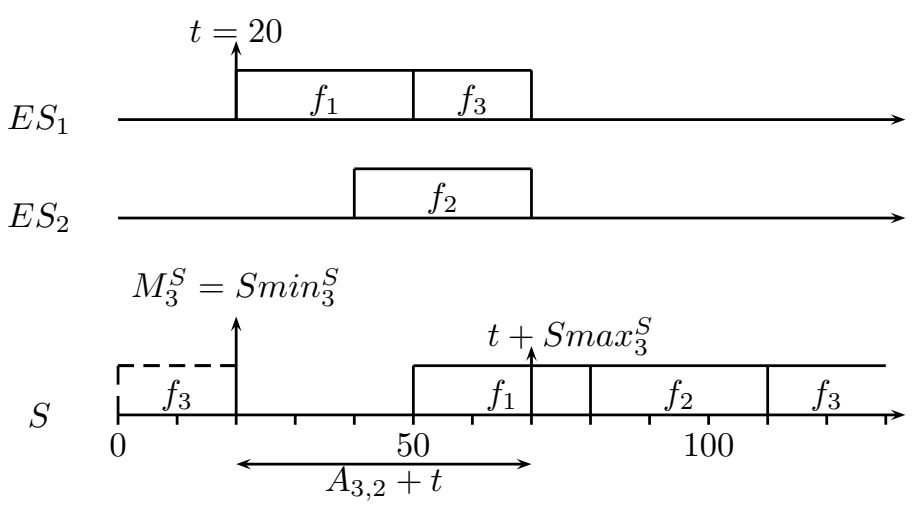

Fig. 3. Understanding the standard notations for the trajectory approach

terms. Combining these terms allows us to calculate, for a specific frame $f_{i}$ released at time $t$, of a flow $v_{i}$, the latest starting time $W_{i, t}^{\text {last }_{i}}$ from its last visited output port last $_{i}$, corresponding to the last node of the network belonging to $\mathcal{P}_{i}$. We have the following expression :

$$
\begin{aligned}
W_{i, t}^{\text {last }_{i}}= & \sum_{\substack{j \in\{1, \ldots, n\} \\
\mathcal{P}_{i} \cap \mathcal{P}_{j} \neq \emptyset}}\left(1+\left\lfloor\frac{t+A_{i, j}}{T_{j}}\right\rfloor\right)^{+} \cdot C_{j} \\
& +\sum_{\substack{h \in \mathcal{P}_{i} /\left\{\text { last }_{i}\right\} \\
\text { s. }}}\left(\max _{\substack{j \in\{1, \ldots, n\} \\
h \in \mathcal{P}_{j}}}\left(C_{j}\right)\right) \\
& +\left(\left|\mathcal{P}_{i}\right|-1\right) \cdot s l \\
& -C_{i}
\end{aligned}
$$

- Term 2 is the delay due to competing flows which delay $v_{i}$ along its trajectory as well as the transmission delay generated by $v_{i}$ itself.

$A_{i, j}$ enters in the composition of the term $(1+$ $\left.\left\lfloor\frac{t+A_{i, j}}{T_{j}}\right\rfloor\right)^{+}$which is the maximal number of frames generated by a flow $v_{j}$ that can delay flow $v_{i}$

To evaluate the end-to-end delay of a flow $v_{i}$, we need the value of $A_{i, j}$ for all encountered flow $v_{j}$. To do this, we first calculate the two terms $\operatorname{Smax}_{i}^{h}$ and $M_{i}^{h}$ which are the same for each flow $v_{j}$. Respectively, $M_{i}^{h}$ is the earliest arrival time of the first packet which delays $i$ on a node $h$, and $\operatorname{Smax}_{i}^{h}$ (see Figure 3).

- Term 3 is the transition cost from one busy period to the next one. When each frame sequence is transmitted from one busy period to the following one, there is a transition delay which is taken as the largest frame transmission time in the frame sequence. For example, in Figure 4 the frame sequence at node $E S_{1}$ is composed by frames $f_{0}$ and $f_{1}$. When they are transmitted to node $S$, the transmission time of frame $f_{0}$ is taken as the transition cost since it is larger than that of frame $f_{1}$.

- $\quad$ Term 4 is the switching latencies along the considered path, considered as a worst-case constant, identical for each link. For each encountered node we add a switching latency to the end-to-end delay of a message $f_{i}$, corresponding to electronic delay. So, in an entire network, the induced latency corresponding 
to $f_{i}$ corresponds to $s l$, multiplied by the number of encountered nodes, represented by $\left|\mathcal{P}_{i}\right|$. As we consider the path from the first switch to the last one of the network, we need to minus our end-to-end delay of $1 * s l$ : given the point that we want to evaluate the end-to-end delay of a flow in a network, we can focus on the delay between its entry point and the output of the last switch in the network. In figure 1, this latency corresponds to the delay between $S_{2}$ and $E S_{6}$.

- Term 5 is subtracted because $W_{i, t}^{\text {last }_{i}}$ is the latest starting time at last $_{i}$. Indeed, $W_{i, t}^{\text {last }_{i}}$ corresponds to the delay between time $\mathrm{t}$ and the beginning of the transmission of $i$ in last $_{i}$ (and not the end of the transmission time). So, the value of $C_{\text {last }}$ needs to be subtracted from the value of $W_{i, t}^{\text {last }_{i}}$.

From the latest starting time, the worst-case end-to-end delay upper bound of the flow $v_{i}$ calculated by the Trajectory approach is obtained by [14]:

$$
R_{i}=\max _{0 \leq t \leq \mathcal{B}_{i}}\left\{W_{i, t}^{\text {last }_{i}}+C_{i}-t\right\}
$$

where $\mathcal{B}_{i}=\sum_{\substack{j \in \llbracket 1, n \rrbracket \\ \mathcal{P}_{i} \cap \mathcal{P}_{j} \neq \emptyset}}\left\lceil\frac{\mathcal{B}_{i}}{T_{j}}\right\rceil \cdot C_{j}$. (see Figure 4)

$B_{i}$ represents the largest possible length of the busy period in each encountered node of the network.

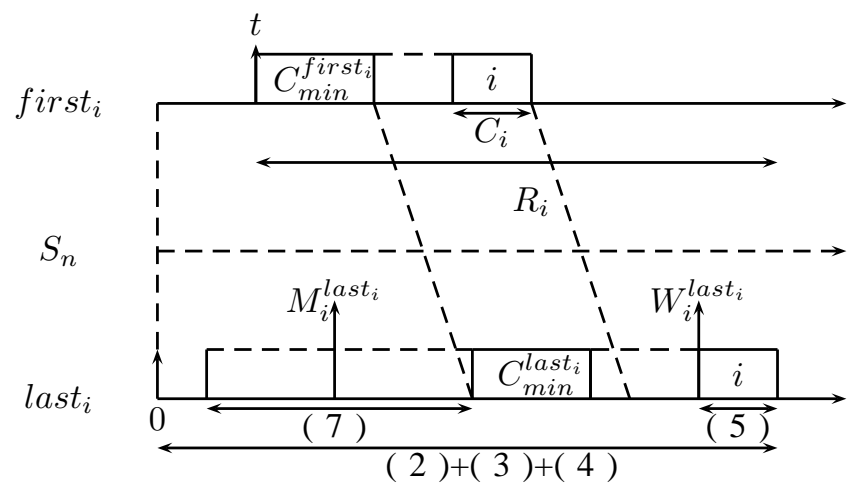

Fig. 4. Calculating the latest starting time of $i$ with the trajectory approach

\section{The serialization of frames}

The classical Trajectory approach considers that competing frames of different flows can arrive at the output port at the same time. However, for frames transmitted from the same input link, their transmissions are necessarily serialized (physical constraint) and they cannot arrive at the output port at the same time. In the following paragraphs, the serialization effect and its solution to the Trajectory approach proposed in [8] are briefly recalled.

The frame serialization exists at each switch $h$ as illustrated in Figure 5. Flows crossing an output port $h$ are transmitted from $k_{h}+1$ input links $\operatorname{IP}_{k}^{h}\left(k \in\left\{0, \ldots, k_{h}\right\}\right)$. For a frame $f_{i}$ of flow $v_{i}$, it crosses $h$ from the input link $\operatorname{IP}_{0}^{h}$, which is the output link $\mathrm{OP}^{h-1}$, to the output link $\mathrm{OP}^{h}$. There are other $k_{h}$ input links $\operatorname{IP}_{k}^{h}\left(k \in\left\{1, \ldots, k_{h}\right\}\right)$ which transmit competing frames to the output link $\mathrm{OP}^{h}$. These frames can delay frame $f_{i}$ in the busy period $b p^{h}$.

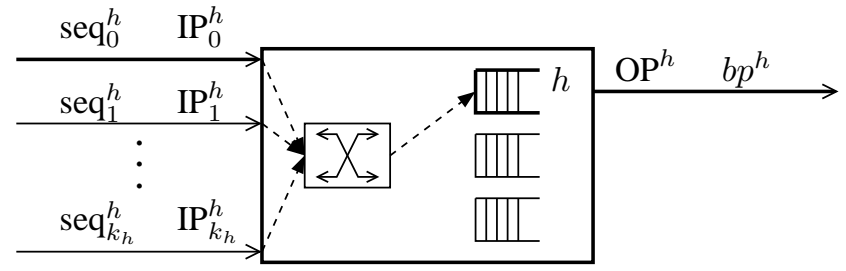

Fig. 5. Illustration on an output link $\mathrm{OP}^{h}$ and $k_{h}+1$ input link $\mathrm{IP}_{k}^{h}$

In [8], an optimization on the Trajectory approach has been proposed by taking into account the serialization effect. An illustration of the optimization is shown in Figure 6. Due to the FIFO scheduling at the output buffer of $h$, any frame arriving later than the arrival time of frame $f_{i}(\theta$ in Figure 6) at node $h$ cannot delay $f_{i}$. Then the delay of $f_{i}$ is maximized by postponing the last frame arrival of each frame sequence from each input link $\operatorname{IP}_{k}^{h}\left(k \in\left\{1, \ldots, k_{h}\right\}\right)$ till the time $\theta$. It has been illustrated in [8] that at the output port $\mathrm{OP}^{h}$, there can be frames transmitted before the first frame arrival from $\mathrm{IP}_{0}^{h}$. These frame transmissions do not delay frame $f_{i}$ and should be taken into account in the delay computation. The associated duration is denoted by $\Delta_{i, t}^{h}$.

From [8], the value of $\Delta_{i, t}^{h}$ is minimized when the first frame of $\mathrm{IP}_{0}^{h}$ is the smallest frame transmitted by $\mathrm{IP}_{0}^{h}$ and the first frame of $\mathrm{IP}_{k}^{h}$ is the largest frame transmitted by the corresponding input link $\mathrm{IP}_{k}^{h}$. The scenario is illustrated in Figure 6 and the computation is given by:

$$
\begin{aligned}
\Delta_{i, t}^{h} & =\max _{x \in\left\{1, \ldots, k_{h}\right\}}\left\{\sum_{v_{j} \in \mathbb{P}_{x}^{h}}\left(\left\lfloor 1+\frac{t+A_{i, j}}{T_{j}}\right\rfloor \cdot C_{j}\right)-\max _{v_{j} \in \mathbb{P}_{x}^{h}}\left(C_{j}\right)\right\} \\
& \left.-\sum_{v_{j} \in \mathbb{P}_{0}^{h}}\left(\left\lfloor 1+\frac{t+A_{i, j}}{T_{j}}\right\rfloor \cdot C_{j}\right)-\min _{v_{j} \in \mathbb{P}_{0}^{h}}\left(C_{j}\right)\right)
\end{aligned}
$$

Then serialization factor at each visited output port along the studied path $\mathcal{P}_{i}$ is given by:

$$
\sum_{h \in \mathcal{P}_{i} /\left\{\text { frst }_{i}\right\}}\left(\Delta_{i, t}^{h}\right)
$$

From [8], it has been shown that $W_{i}^{\text {last }_{i}}$ including the serialization factors is given by:

$$
\begin{aligned}
& W_{i, t}^{\text {last }_{i}}=\sum_{\substack{j \in\{1, \ldots, n\} \\
\mathcal{P}_{i} \cap \mathcal{P}_{j} \neq \emptyset}}\left(1+\left\lfloor\frac{t+A_{i, j}}{T_{j}}\right\rfloor\right)^{+} \cdot C_{j} \\
& +\sum_{h \in \mathcal{P}_{i} /\left\{\text { last }_{i}\right\}}\left(\max _{\substack{j \in\{1, \ldots, n\} \\
h \in \mathcal{P}_{j}}}\left(C_{j}\right)\right) \\
& +\left(\left|\mathcal{P}_{i}\right|-1\right) \cdot s l \\
& -\sum_{h \in \mathcal{P}_{i} /\left\{\text { first }_{i}\right\}}\left(\Delta_{i, t}^{h}\right) \\
& -C_{i}
\end{aligned}
$$




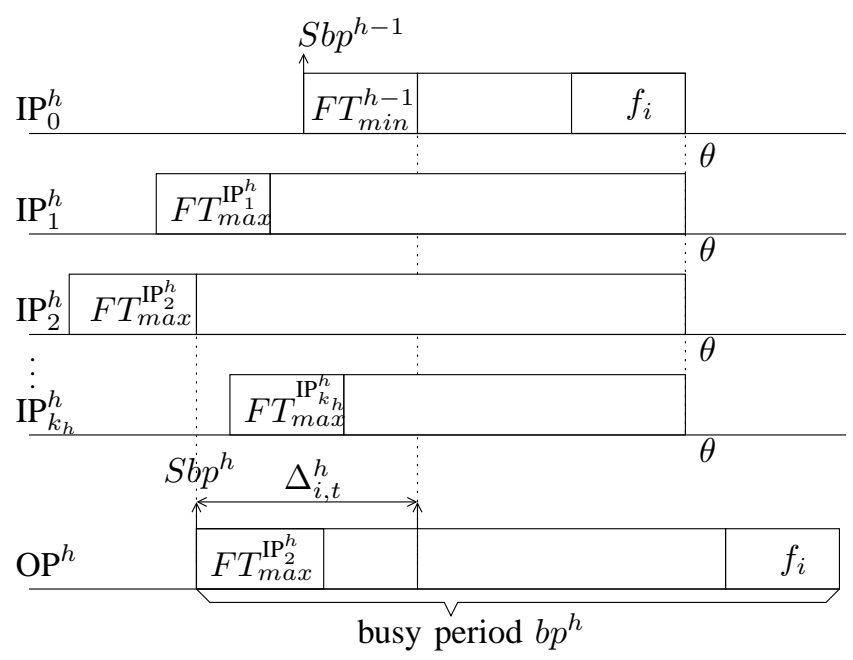

Fig. 6. Illustration of term $\Delta_{i, t}^{h}$

This formula has been shown to be optimistic in particular case [14]. We will now illustrate why it can be optimistic.

\section{PROBLEM: COUNTER-EXAMPLE WITH OPTIMISM}

The Trajectory approach considers the worst-case scenario that can happen to a frame along its trajectory in order to guarantee the delay upper bound computation. Recently, a counter-example has been shown in [14] to point out that for some corner cases, the Trajectory approach can lead to optimistic computed delay upper bounds. This example is given in Figure 7.

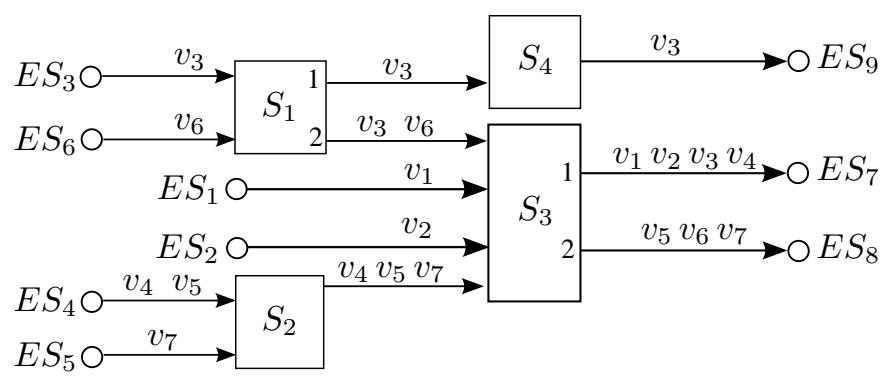

Fig. 7. An illustrative AFDX example

It means that the exact worst-case delay of a frame can be larger than the one obtained with the current state-of-theart Trajectory approach. For example, for frame $f_{1}$ of flow $v_{1}$, which can be delayed by frames of flows $v_{2}, v_{3}, v_{4}$ at the output of switch $S_{3}$, its exact worst-case delay is $180 \mu \mathrm{s}$ as shown in Figure 8. However, the delay upper bound of frame $f_{1}$ computed by the Trajectory approach is $160 \mu \mathrm{s}$, which is lower than the exact worst-case delay. Therefore the Trajectory approach gives an optimistic result (20 $\mu s$ of pessimism) in this case.

The Trajectory approach considers all the possible values of time $t$ in the time interval $\left[0, B_{i}\right]$. Time $t$ corresponds to the arrival time of frame $f_{i}$ at its source node first $_{i}$, and each value of $t$ corresponds to a release time scenario for frame $f_{i}$ that must be analyzed. Therefore, the Trajectory approach searches a time $t$ in a given time interval leading to the maximum value of $R_{i}$ in Formula 6, which corresponds to the worst-case scenario. In the example, the computed delay upper bound $R_{1}$ is obtained when $t=0$. The corresponding scenario is illustrated in Figure 9.

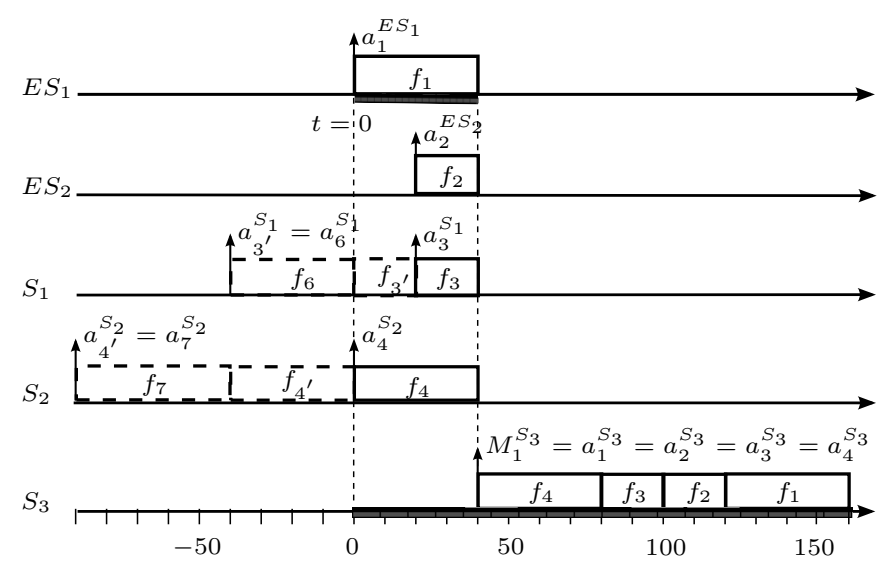

Fig. 9. Delay of frame $f_{1}$ computed by the Trajectory approach

In the case of frame $f_{1}$, since it is the only frame transmitted from $E S_{1}$, we have $M_{1}^{S_{3}}=t+S_{\text {max }_{1}}^{S_{3}}=40 \mu \mathrm{s}$, giving the interval $\left[M_{1}^{S_{3}}, t+S_{\text {max }_{1}}^{S_{3}}\right]$ which leads to only a time instant $40 \mu \mathrm{s}$. It means that for flows $v_{2}, v_{3}$ and $v_{4}$ competing with flow $v_{1}$ at the output port of $S_{3}$ (port 1 in Figure 7), only the frames arriving at this output port at time $t=40 \mu \mathrm{s}$ can delay frame $f_{1}$. Therefore, for each flow there is at most one frame that can delay frame $f_{1}$, among frame $f_{2}$, frame $f_{3}$ and frame $f_{4}$ as shown in Figure 9.

However, as shown in Figure 8, frames $f_{3^{\prime}}$ and $f_{4^{\prime}}$ which can actually delay frame $f_{1}$ are not taken into account since they arrive before time $M_{1}^{S_{3}}=40 \mu \mathrm{s}$. Therefore, the interval $\left[M_{1}^{S_{3}}, t+S_{\text {max }_{1}}^{S_{3}}\right]$ is not correct (under-estimated). Indeed, in the example $t=0$ does not lead to the worst-case scenario.

Actually, for $t=40 \mu s$, the Trajectory approach computes a longer interval $\left[M_{1}^{S_{3}}, t+S_{\text {max }_{1}}^{S_{3}}\right]=[40,80]$ as shown in Figure 10. In this case, both frames $f_{3}$ and $f_{3^{\prime}}$ as well as frames $f_{4}$ and $f_{4^{\prime}}$ are taken into account in the computation. However, the computation still gives an optimistic result which is $R_{1}(t=40)=140 \mu s$.

The computation subtracts a serialization factor $\Delta_{1}^{S_{3}}=$ $40 \mu s$ (Term 7) since the transmission of frame $f_{4^{\prime}}$ at $S_{3}$ does not delay the frame $f_{1}$ due to the serialization effect. Meanwhile, a time $t=40 \mu \mathrm{s}$ is also subtracted in Formula 6 since frame $f_{1}$ arrives at time $t$ and therefore the time interval $[0, t]$ is not part of the delay of frame $f_{1}$. In fact, these two parts of subtractions overlap and the same time interval is subtracted twice from the computation (detailed explanation will be given in the next section). It then results in an optimistic computed delay $140 \mu s$ of frame $f_{1}$, as illustrated in Figure 10.

In the next paragraphs, a detailed analysis of the optimism will be given and a solution to the optimism problem will be proposed. 


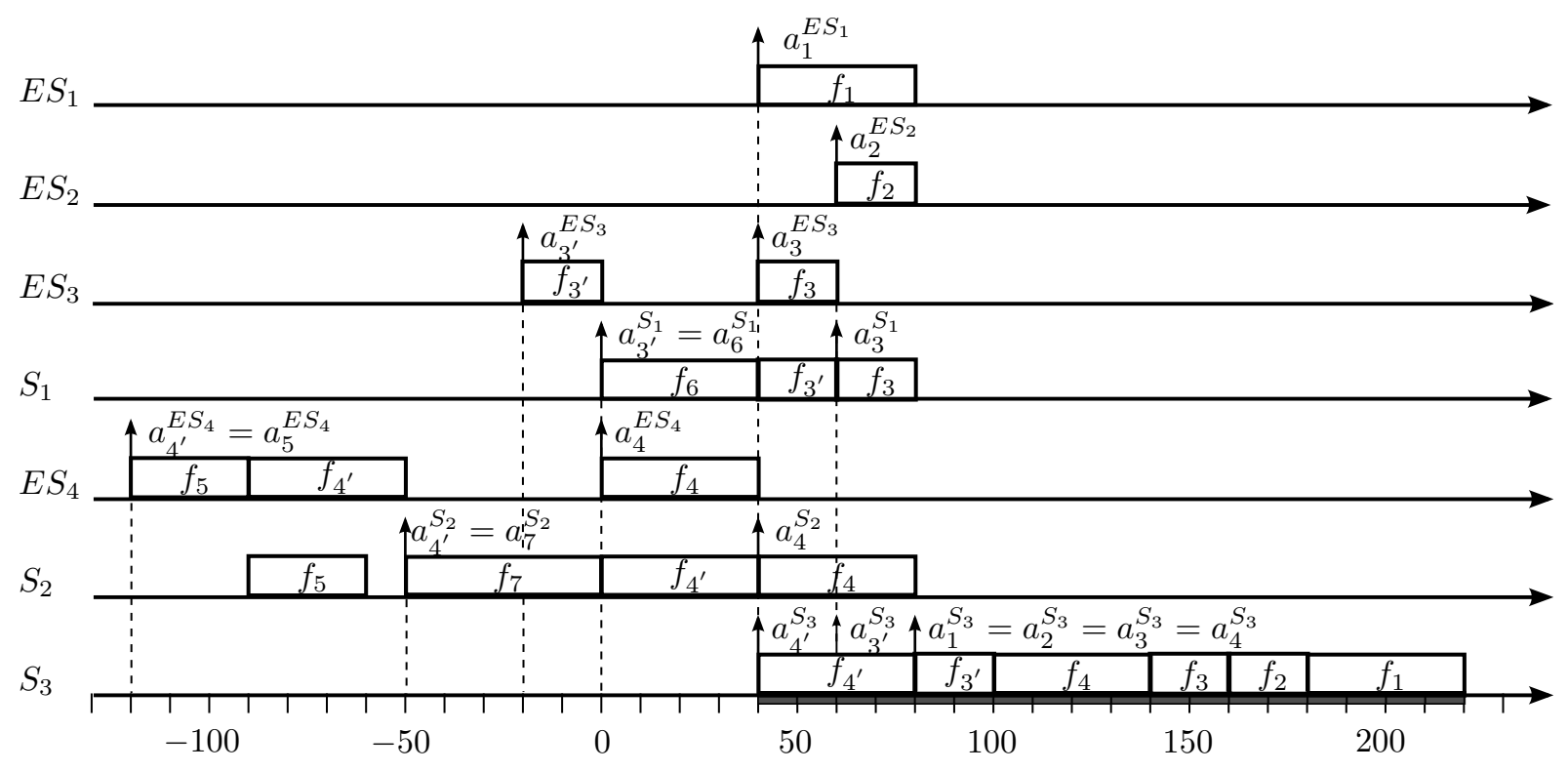

Fig. 8. Worst-case ETE delay of frame $f_{1}$ at time $t=40$

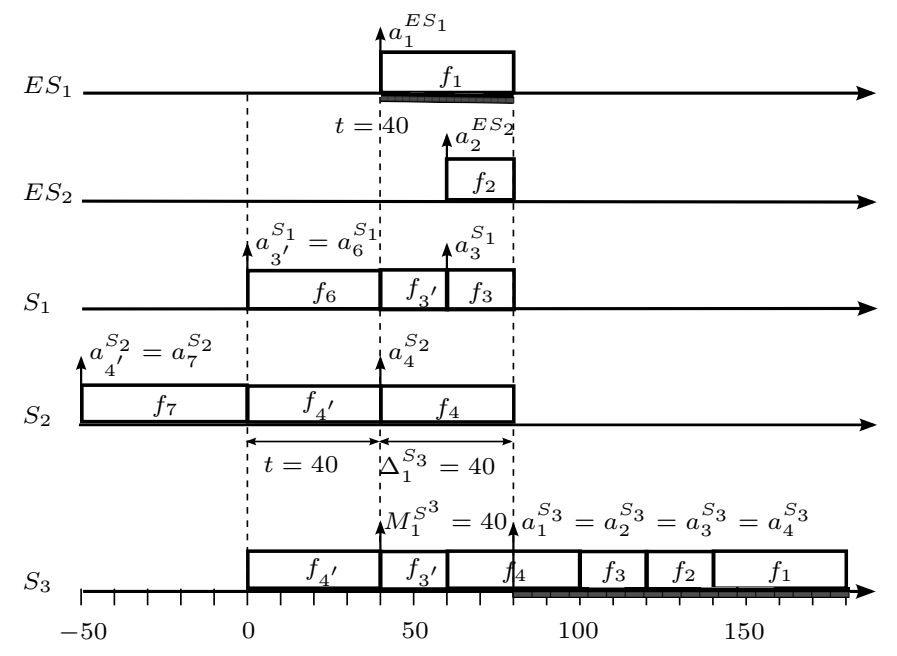

Fig. 10. Delay of frame $f_{1}$ computed by the Trajectory approach at time $t=40 \mu s$

\section{A CORRECTION OF THE TRAJECTORY APPROACH}

As shown in the previous paragraphs, for a frame $f_{i}$ of a flow $v_{i}$ the problem of the under-estimated interval $\left[M_{i}^{h}, t+S_{\text {max }_{i}}^{h}\right]$ disappears with the examinations of all the possible values of $t$. However, at the same time subtracting time $t$ from the delay of frame $f_{i}$ (Equation 6) can overlap with part of the serialization factor (Term 7). The following paragraphs first examine the optimism of the Trajectory approach by an illustrative example, with both the classical approach and the serialization optimization respectively. Then the analysis is developed in the general case and a correction of the optimism problem is proposed.

\section{A. Computation with the classical approach}

In order to explain this problem in details, let us consider the example given in Figure 1. The flow temporal characteris- tics are given in Table I. In the example, the switching latency is considered as null.

\begin{tabular}{|c|c|c|c|c|c|c|c|c|c|}
\hline$v_{i}$ & $v_{1}$ & $v_{2}$ & $v_{3}$ & $v_{4}$ & $v_{5}$ & $v_{6}$ & $v_{7}$ & $v_{8}$ & $v_{9}$ \\
\hline$C_{i}(\mu s)$ & 40 & 40 & 40 & 40 & 40 & 40 & 40 & 40 & 40 \\
$T_{i}(\mu s)$ & 4000 & 120 & 4000 & 320 & 4000 & 4000 & 4000 & 4000 & 4000 \\
\hline
\end{tabular}

TABLE I. FLOW TEMPORAL CHARACTERISTICS OF THE NETWORK EXAMPLE

Frame $f_{1}$ of flow $v_{1}$ is under study. It follows the path $\mathcal{P}_{1}=\left\{E S_{1}, S_{1}, S_{2}\right\}$ and it can be delayed by frames of flows $v_{2}$ and $v_{3}$ at the output port of $S_{1}$ as well as by flows $v_{4}, v_{5}$, $v_{6}, v_{7}$ and $v_{8}$ at the output port of $S_{2}$.

One worst-case scenario of frame $f_{1}$ is given in Figure 11 when $t=120 \mu \mathrm{s}$. The worst-case delay of frame $f_{1}$ is $320 \mu \mathrm{s}$. As illustrated in this figure, flow $v_{2}$ has two frames $f_{2}$ and $f_{2^{\prime}}$ delaying frame $f_{1}$ at the output port of $S_{1}$, and flow $v_{4}$ has two frames $f_{4}$ and $f_{4^{\prime}}$ delaying frame $f_{1}$ at the output port of $S_{1}$.

Let us now consider the Trajectory approach. Since flow $v_{1}$ is the only flow emitted by $E S_{1}$, then $M_{1}^{S_{1}}=40 \mu \mathrm{s}$ and $S_{\text {max }_{1}}^{S_{1}}=S_{\text {min }_{1}}^{S_{1}}=C_{1}=40 \mu \mathrm{s}$. First, for each flow encountered by $v_{1}$, we need to calculate the value of $A_{i, j}$. For this, we apply the Equation 1 . Flow $v_{2}$ is emitted by $E S_{2}$ and can be delayed by frames of flow 9 and then it can be delayed by frames of flow $v_{3}$ at the output port of $S_{3}$, which gives $S_{\max _{2}}^{S_{1}}=160 \mu \mathrm{s}$ and $S_{\text {min }_{2}}^{S_{1}}=80 \mu \mathrm{s}$. Therefore, the computation gives $A_{1,2}$ as follows:

$$
\begin{aligned}
A_{1,2} & =S_{\max _{1}}^{S_{1}}-S_{\min _{2}}^{S_{1}}-\left(M_{1}^{S_{1}}-S_{\max _{2}}^{S_{1}}\right) \\
& =40-80-(40-160) \\
& =80 \mu \mathrm{s}
\end{aligned}
$$

In the same way, the values of $A_{1,3}=40 \mu \mathrm{s}$ and $A_{1,4}=$ $A_{1,5}=A_{1,6}=A_{1,7}=A_{1,8}=200 \mu \mathrm{s}$ are obtained. So, we obtain the following results : 


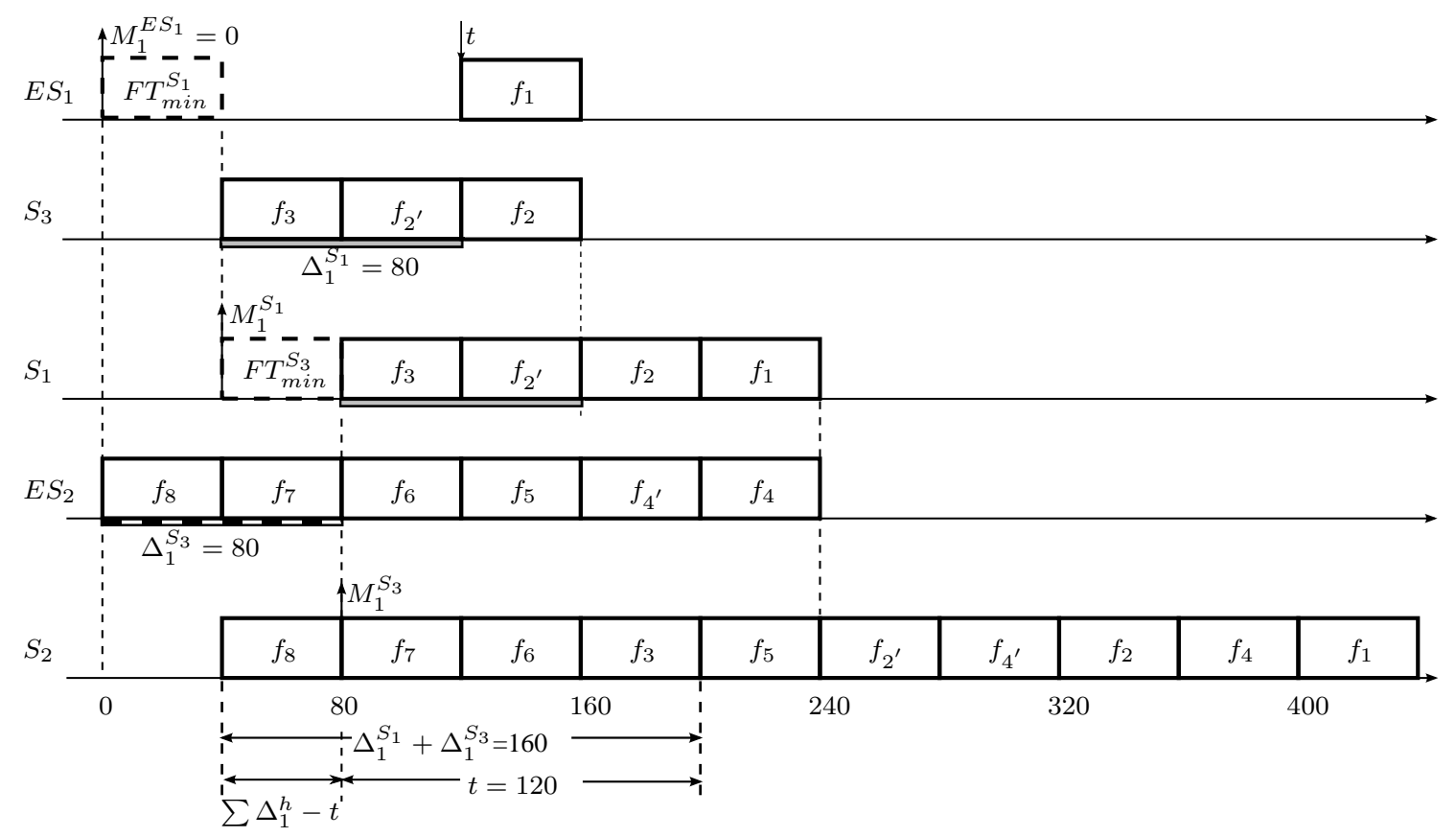

Fig. 11. Worst-case scenario of frame $f_{1}$

\begin{tabular}{|c|c|c|c|c|c|c|c|c|c|}
\hline$j$ & 1 & 2 & 3 & 4 & 5 & 6 & 7 & 8 & 9 \\
\hline$A_{1, j}$ & 0 & 40 & 200 & 200 & 200 & 200 & 200 & 200 & 200 \\
\hline & TABLE II. VALUES OF $A_{i, j}$ FOR EACH FLOW
\end{tabular}

Therefore at time $t=120 \mu \mathrm{s}$, the $W_{1,120}^{S_{2}}$ is given by:

$$
\begin{aligned}
W_{1,120}^{S_{2}}= & \sum_{j \in\{1, \ldots, 8\}}\left(1+\left\lfloor\frac{120+A_{i, j}}{T_{j}}\right\rfloor\right)^{+} \cdot C_{j} \\
& +\sum_{h \in\left\{E S_{1}, S_{1}\right\}}\left(\max _{\substack{j \in\{1, \ldots, 8\} \\
h \in \mathcal{P}_{j}}}\left(C_{j}\right)\right) \\
& -\sum_{h \in\left\{S_{1}, S_{2}\right\}}\left(\Delta_{1,120}^{h}\right) \\
& -C_{1}{ }\left(1+\left\lfloor\frac{120}{4000}\right\rfloor\right)^{+} \cdot 40+\left(1+\left\lfloor\frac{120+80}{120}\right\rfloor\right)^{+} \cdot 40 \\
& +\left(1+\left\lfloor\frac{120+40}{4000}\right\rfloor\right)^{+} \cdot 40+\left(1+\left\lfloor\frac{120+200}{320}\right\rfloor\right)^{+} \cdot 40 \\
& +\left(1+\left\lfloor\frac{120+200}{4000}\right\rfloor\right)^{+} \cdot 40 \times 4 \\
& +40+40-\Delta_{1,120}^{S_{1}}-\Delta_{1,120}^{S_{2}}-40 \\
& 440-\Delta_{1,120}^{S_{1}}-\Delta_{1,120}^{S_{2}}
\end{aligned}
$$

First, let us consider the classical Trajectory approach without serialization factor. In this case, the $W_{1,120}^{S_{2}}=440 \mu \mathrm{s}$ and then $R_{1}=W_{1,120}^{S_{2}}+C_{1}-120=360 \mu \mathrm{s}$, which is pessimistic compared to the worst-case delay $320 \mu \mathrm{s}$ in Figure 11. This scenario is illustrated in Figure 12. The pessimism is generated because the computation is done based on pessimistic assumption that all the frames of different flows arrive at the same time even if they are serialized by some input link (the serialization factor is not considered).

It has been illustrated in Figure 12 that although frames $f_{4^{\prime}}, f_{5}, f_{6}, f_{7}$ and $f_{8}$ are considered arriving at the same time, frames $f_{6}, f_{7}$ and $f_{8}$ do not delay frame $f_{1}$. Indeed, the

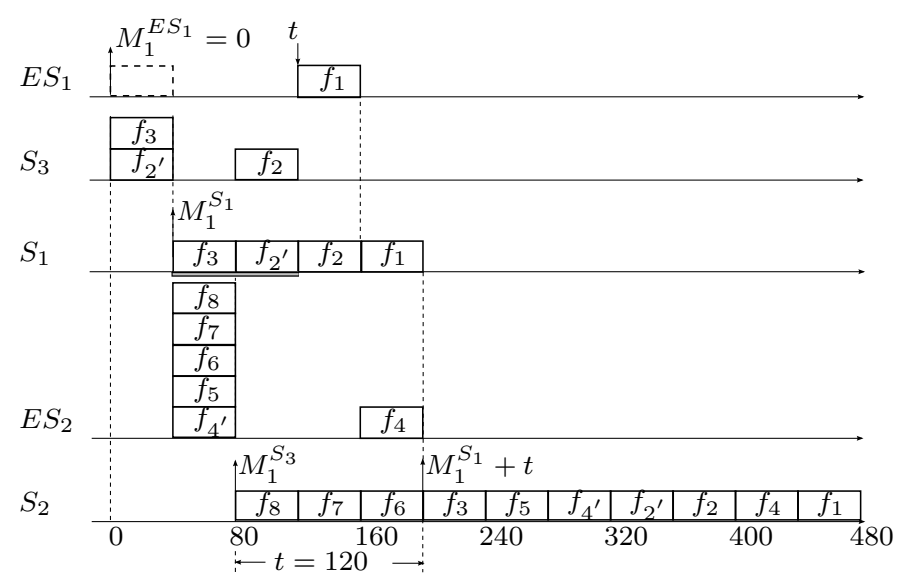

Fig. 12. One worst-case scenario of frame $f_{1}$ considered by the classical Trajectory approach

computation of $W_{1}^{S_{2}}$ is based on the maximized busy period $b p^{S_{2}}$ which starts at time $M_{1}^{S_{2}}$ (shown in Figure 12). Since frame $f_{1}$ arrives at $E S_{1}$ at time $t$, there is a time interval of $t$ which does not contribute to the delay of frame $f_{1}$. It means that during the busy period $b p^{S_{2}}$, the frame transmission during $\left[M_{1}^{S_{2}}, M_{1}^{S_{2}}+t\right]$ do not delay frame $f_{1}$, which are exact the frames $f_{6}, f_{7}$ and $f_{8}$ in the example.

Suppose that the delay upper bound of frame $f_{i}$ is obtained when $f_{i}$ arrives at first $_{i}$ at a given time $t$. The corresponding scenario of $R_{i}$ is illustrated by the first bar in Figure 13 where the time interval $t$ is subtracted since it is not part of delay. Then the shadow part in the bar represents the delay $R_{i}$.

Since the classical Trajectory approach considers that the competing frames arrive after time $M_{i}^{h}$ at each node $h$ and there is no frame transmitted before $M_{i}^{h}$, then the subtrac- 


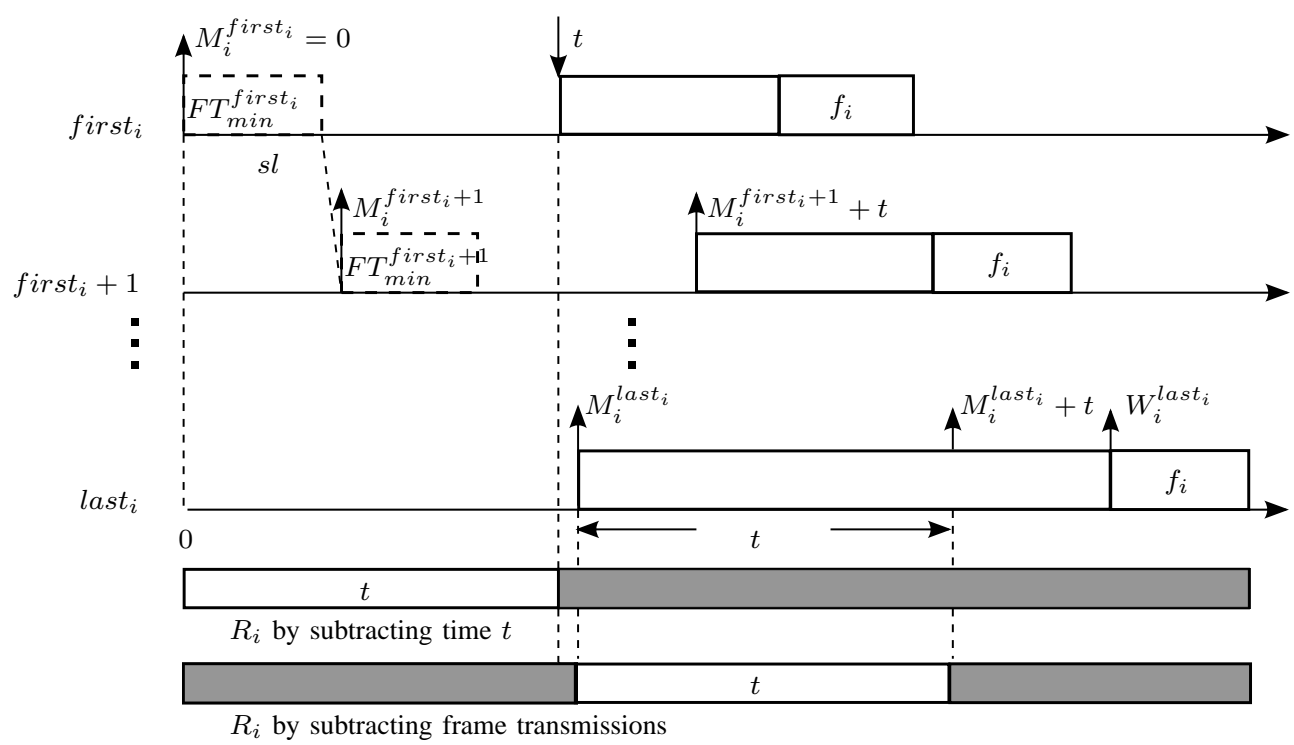

Fig. 13. General scenario of frame $f_{i}$ of the classical Trajectory approach

tion of the time interval $t$ in Formula 6 is equivalent to the subtraction of the WCTT of frames transmitted between $\left[M_{i}^{\text {last }_{i}}, M_{i}^{\text {last }_{i}}+t\right]$ at the last visited node last $_{i}$. The corresponding scenario is also illustrated by the second bar in Figure 13 where the time interval $t$ (white bar) is moved between $M_{i}^{\text {last }_{i}}$ and $M_{i}^{\text {last }_{i}}+t$.

\section{B. Computation with Serialization effect}

As explained in Section III-C, frames transmitted from the same input link are serialized and cannot arrive at the output at the same time. Therefore, the scenario shown in Figure 12 where frames $f_{4^{\prime}}, f_{5}, f_{6}, f_{7}$ and $f_{8}$ arrive at $S_{2}$ at the same time is impossible and pessimistic.

Indeed, as shown in Figure 11, at the output port of $S_{1}$, frames $f_{2}, f_{2^{\prime}}$ and $f_{3}$ arrive from the same link $\left\{S_{3}, S_{1}\right\}$. Since frame $f_{1}$ is the only frame transmitted from $E S_{1}$, only one frame from the input link $\left\{S_{3}, S_{1}\right\}$ can delay frame $f_{1}$. In this illustration, it is frame $f_{2}$. Then the serialization factor is computed by $\Delta_{1,120}^{S_{1}}=C_{3}+C_{2}=80 \mu \mathrm{s}$. Similarly, at the output port of $S_{2}$, the serialization factor is computed by $\Delta_{1,120}^{S_{2}}=80 \mu \mathrm{s}$. Then by taking into account the serialization effect, we have $W_{1,120}^{S_{2}}=440-\Delta_{1,120}^{S_{1}}-\Delta_{1,120}^{S_{2}}=280 \mu \mathrm{s}$ and $R_{1}(120)=W_{1,120}^{S_{2}}+C_{1}-t=200 \mu \mathrm{s}$, which is optimistic compared to the worst-case delay $320 \mu \mathrm{s}$ in Figure 11.

The optimism is introduced since the subtraction of serialization factors is partially overlapped with the subtraction of the time interval $t$ in Equation 6. The classical approach subtracts the transmissions of frames $f_{6}, f_{7}$ and $f_{8}$ by the subtraction of time $t$ as shown in Figure 12, while the serialization factors subtract the transmissions of frames $f_{3}, f_{6}$, $f_{7}$ and $f_{8}$ as illustrated in Figure 11. In this case, the frames $f_{6}$, $f_{7}$ and $f_{8}$ are actually subtracted twice and therefore leading to the optimistic result.

In the general case, the serialization factors subtract frame transmissions of $\sum_{\substack{h \in \mathcal{P}_{i} \\ h \neq \text { first }_{i}}} \Delta_{i, t}^{h}$ from the computation at the last visited output last $_{i}$. For the purpose of illustration, we first consider the scenario in Figure 6. The starting instant of the busy period $b p^{h}$ is denoted $S b p^{h}$ as shown in Figure 6. Since the input $\mathrm{IP}_{0}^{h}$ is the output busy period $b p^{h-1}$ in the previous output port $h-1$, then $S b p^{h-1}$ is also indicated in Figure 6 . Therefore, we have:

$$
S b p^{h}=S b p^{h-1}+F T_{\min }^{h-1}+s l-\Delta_{i, t}^{h}
$$

At the source node first $_{i}$, the worst-case scenario is when all the frames arrive at the same time as frame $f_{i}$ and delay $f_{i}$. Then $S b p^{\text {first }_{i}}$ is equal to the arrival time of $f_{i}$, i.e. $S b p^{\text {first }_{i}}=t$. Based on the Equation 8, the computation propagates till last $_{i}$ where we have:

$$
\begin{aligned}
S b p^{\text {last }_{i}} & =t+\sum_{\substack{h \in \mathcal{P}_{i} \\
h \neq \text { last }_{i}}}\left(F T_{\text {min }}^{h}+s l\right)-\sum_{\substack{h \in \mathcal{P}_{i} \\
h \neq \text { irst }_{i}}} \Delta_{i, t}^{h} \\
& =t+M_{i}^{\text {last }_{i}}-\sum_{\substack{h \in \mathcal{P}_{i} \\
h \neq \text { first }_{i}}} \Delta_{i, t}^{h}
\end{aligned}
$$

This scenario is illustrated in Figure 14 which shows that at the last visited output last $_{i}$, the frame transmissions between $S b p^{\text {last }_{i}}$ to $t+M_{i}^{\text {last }_{i}}$ are subtracted from the computation.

In the given example, we want to evaluate the error rate induced by the optimism in the Trajectory approach(compared to the standard hand-built method). So, we make the following evaluation :

$$
E(\text { ErrorRate })=(320-280) * 100 / 320=12,5 \% .
$$

In this simple example, the optimism induced represents more than $10 \%$ of the result. In strong-constrained domains concerned by network and real time, like aeronautics or public transports, which have industrial very-high needs in terms of 


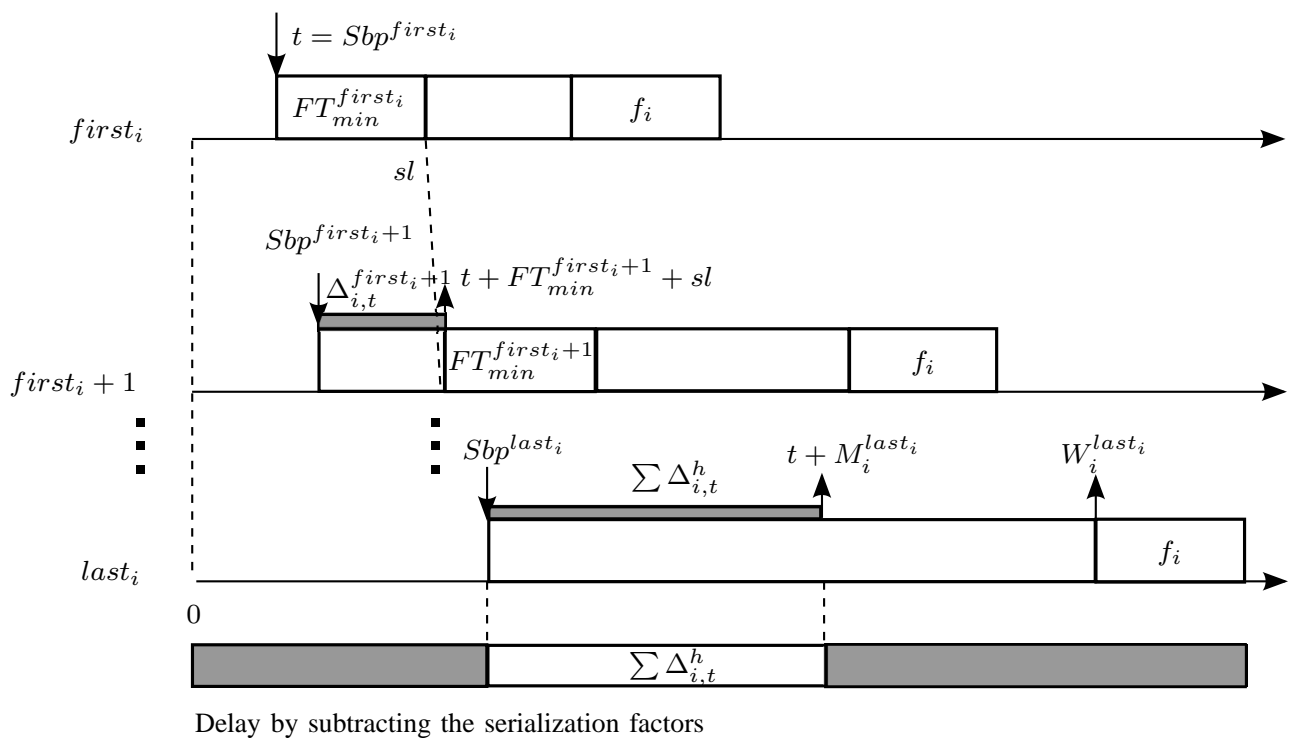

Fig. 14. General scenario of frame $f_{i}$ considered by the Trajectory approach with serialization effect

reliability and accuracy, such a high error rate directly corresponds to invalidating the method of the Trajectory approach. That is why we need to find a solution to this optimism.

\section{A solution to optimism}

Let us focus on the comparison between Figure 13 where a time $t$ is subtracted from $M_{i}^{\text {last }}$ to $M_{i}^{\text {last }_{i}}+t$ and Figure 14 where the serialization factors are subtracted from $S b p^{\text {last }_{i}}$ to $M_{i}^{\text {last }_{i}}+t$. These exists an overlapped time interval $\left[\max \left(M_{i}^{\text {last }_{i}}, S b p^{\text {last }_{i}}\right), M_{i}^{\text {last }_{i}}+t\right]$ which is subtracted twice in the computation of delay upper bounds, and therefore leading to the optimistic results. In the example, this time interval is $[80,200]$ as shown in Figure 11.

One solution to solve this problem is to take into account the subtraction of time $t$ in the serialization factors. More precisely, instead of subtracting the time of frame transmissions before time $t+M_{i}^{\text {last }}{ }_{i}$, consider to subtract it at the last visited node the frame transmissions before $M_{i}^{\text {last }}{ }_{i}$ since only this part is not considered by the classical Trajectory approach.

Theorem 1. The serialization effect in the Trajectory approach is taken into account by a serialization correction:

$$
\left(\sum_{\substack{h \in \mathcal{P}_{i} \\ h \neq \text { first }_{i}}}\left(\Delta_{i, t}^{h}\right)-t\right)^{+}
$$

Proof: The serialization correction considers the serialization effect by taking the difference between the serialization factors and the subtracted time $t$. More precisely, two cases are considered and illustrated below.

First, we considers the case $\sum_{\substack{h \in \mathcal{P}_{i} \\ h \neq \text { last }_{i}}}\left(\Delta_{i, t}^{h}\right) \leq t$, and the corresponding scenario is illustrated in Figure 15. Since the overlapped time interval $\left[S b p^{\text {last }_{i}}, M_{i}^{\text {last }_{i}}+t\right]$ is covered by the time interval $\left[M_{i}^{\text {last }_{i}}, M_{i}^{\text {last }_{i}}+t\right]$, the serialization effect is taken into account by subtracting time $t$ in Formula 6 . Therefore, the serialization correction is taken as value 0 .

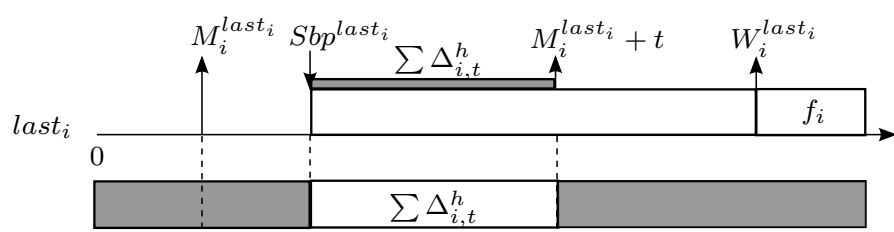

Delay by: subtracting the serialization façtors

\begin{tabular}{|l|l|l|}
\hline & $t$ & \\
\hline
\end{tabular}

$R_{i}$ by subtracting time $t$

Fig. 15. Scenario of $\sum \Delta_{i, t}^{h} \leq t$

Second, we consider the case $\sum_{\substack{h \in \mathcal{P}_{i} \\ h \neq \text { last }_{i}}}\left(\Delta_{i, t}^{h}\right)>t$, and the corresponding scenario is illustrated in Figure 16. The frame transmissions before $M_{i}^{\text {last }_{i}}$ do not delay frame $f_{i}$ and their time needs to be subtracted, which corresponds to the serialization correction. The rest part of the serialization factors is equal to the overlapped time interval $\left[M_{i}^{\text {last }_{i}}, M_{i}^{\text {last }_{i}}+t\right]$ and it is subtracted in Formula 6.

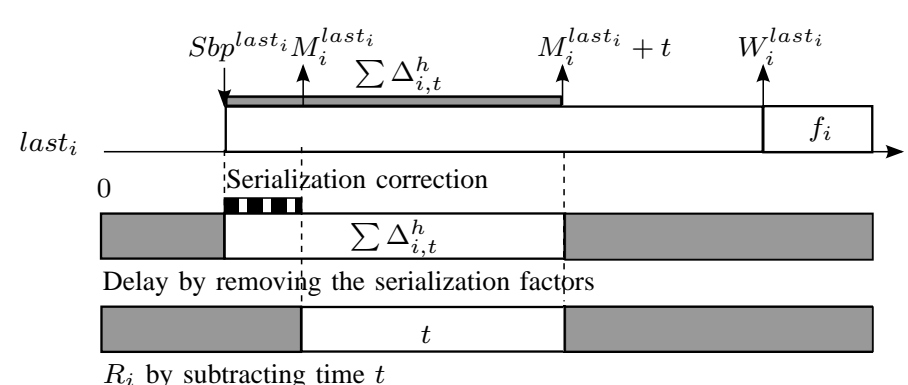

Fig. 16. Scenario of $\sum \Delta_{i, t}^{h}>t$

Therefore, the Term 9 is a correction of Term 7 of serialization factors. 
As it has been demonstrated, the overlapped time interval $\left[\max \left(M_{i}^{\text {last }_{i}}, S b p^{\text {last }_{i}}\right), M_{i}^{\text {last }_{i}}+t\right]$ is subtracted twice in the computation and it can lead to optimistic result of delay upper bound. The optimism introduced by the Trajectory approach presented in III is then computed by:

$$
\min \left(\sum_{\substack{h \in \mathcal{P}_{i} \\ h \neq \text { first }_{i}}} \Delta_{i, t}^{h}, t\right)
$$

The overlapped time interval is equal to 0 only when $t=0$ or when $\sum_{\substack{h \in \mathcal{P}_{i} \\ h \neq \text { first }_{i}}} \Delta_{i, t}^{h}=0$.

In the example, the value of the serialization correction is given by:

$-\left(\left(\Delta_{1,120}^{S_{1}}+\Delta_{1,120}^{S_{2}}\right)-t\right)^{+}=-((80+80)-120)^{+}=-40 \mu s$

It is illustrated by the transmission time of frame $f_{8}$ in Figure 11. Thus, the $W_{1,120}^{S_{2}}$ computed by the Trajectory approach with the serialization correction is:

$$
W_{1,120}^{S_{2}}=440-40=400 \mu \mathrm{s}
$$

The delay upper bound of frame $f_{1}$ is then obtained by

$$
R_{1}=400+40-120=320 \mu s
$$

which is the exact worst-case delay of frame $f_{1}$ in the example.

\section{CONCLUSION}

In this paper, we have considered the problem of computing worst-case end-to-end delays of flows sent on an AFDX FIFO network. We considered the Trajectory approach, known to provide tight worst-case end-to-end delay upper bounds. Recently, it has been shown that this approach can lead to optimistic end-to-end delays thus leading to certification issues. Our goal was to characterize this optimism problem and to provide a solution to it.

We presented the different sources of optimism in the Trajectory approach on pathological examples, including the underestimation of the release time interval used to compute the worst-case end-to-end delay of a flow and a problem on the computation of the serialization factors for flows sent on the same link. The error rate found in our examples can reach $10 \%$, a significant error. We then solved the optimism problem in the general case. As a perspective, we would like to compute the average error rate for random sets of flows.

\section{REFERENCES}

[1] P. R.Santos, A.Vieira, "Flexible, efficient and robust real-time communication with server-based ethernet switching," Factory Communication Systems (WFCS), 2010 8th IEEE International Workshop, pp. 131-140, 2010.

[2] ARINC 664, ACCE Std. 664, 2002-2008.

[3] W. Steiner and G. Bauer, "Ttethernet dataflow concept," Network Computing and Applications, 2009. NCA 2009., pp. 319-322, 2009.

[4] S. Martin, P. Minet, and L. George, "End-to-end response time with fixed priority scheduling: trajectory approach versus holistic approach," Feb. 2004, pp. 37-56.

[5] M. H. J.J. Gutirrez, J.C. Palencia, "Holistic schedulability analysis for multipacket messages in afdx networks," in Real-Time Systems, Mar. 2014, pp. 230-269.
[6] J.-Y. L. Boudec, "Application of network calculus to guaranteed service networks," IEEE Trans. Inf. Theory, vol. 44, no. 3, pp. 1087-1096, May 1998.

[7] J.-P. Georges, E. Rondeau, and T. Divoux, "Evaluation of switched Ethernet in an industrial context by using the network calculus," in 4th IEEE Int. Workshop on Factory Communication Systems(WFCS), Văsterås, Sweden, Aug. 2002, pp. 19-26.

[8] H. Bauer, J.-L. Scharbarg, and C. Fraboul, "Improving the worst-case delay analysis of an AFDX network using an optimized Trajectory approach,” IEEE Trans. Ind. Informat., vol. 6, no. 4, pp. 521-533, 2010.

[9] S. Martin and P. Minet, "Schedulability analysis of flows scheduled with FIFO: application to the expedited forwarding class," in Proc. IEEE International Parallel and Distributed Processing Symposium (IPDPS), Rhodes Island, Greece, Apr. 2006, pp. 8-pp.

[10] - "Worst case end-to-end response times of flows scheduled with FP/FIFO," in Proc. IEEE Int. Conf. on Networking, Mauritius, Apr. 2006, pp. 54-60.

[11] S. Martin, P. Minet, and L. George, "The Trajectory approach for the end-to-end response times with non-preemptive FP/EDF*," in Software Engineering Research and Applications. Springer, 2006, pp. 229-247.

[12] X. Li, J.-L. Scharbarg, and C. Fraboul, "Analysis of the pessimism of the Trajectory approach for upper bounding end-to-end delay of sporadic flows sharing a switched Ethernet network," in Proc. 19th Int. Conf. on Real-Time and Network and Systems (RTNS), Nantes, France, Sep. 2011.

[13] H. Bauer, J.-L. Scharbarg, and C. Fraboul, "Applying and optimizing trajectory approach for performance evaluation of AFDX avionics network," in Proc. IEEE Emerging Technologies and Factory Automation (ETFA), Marllorca, Spain, Sep. 2009, pp. 1-8.

[14] G. Kemayo, F. Ridouard, H. Bauer, and P. Richard, "Optimistic problem in the trajectory approach in FIFO context," in Proc. IEEE Int. Conf. on Emerging Technologies and Factory Automation (ETFA). IEEE, Sep. 2013, pp. 1-8. 